\title{
GaN-based distributed feedback laser diodes for optical communications
}

Steffan Gwyn, Scott Watson, Shaun Viola, Giovanni Giuliano, Thomas J. Slight, et al.

Steffan Gwyn, Scott Watson, Shaun Viola, Giovanni Giuliano, Thomas J. Slight, Szymon Stanczyk, Szymon Grzanka, Amit Yadav, Kevin E. Docherty, Edik Rafailov, Piotr Perlin, Stephen P. Najda, Mike Leszczynski, Anthony E. Kelly, "GaN-based distributed feedback laser diodes for optical communications," Proc. SPIE 11207, Fourth International Conference on Applications of Optics and Photonics, 1120700 (3 October 2019); doi: 10.1117/12.2527074 (AOP 2019), 2019, Lisbon, Portugal 


\title{
GaN-based Distributed Feedback Laser Diodes for Optical Communications
}

\author{
Steffan Gwyn*a, Scott Watson ${ }^{a}$, Shaun Viola ${ }^{a}$, Giovanni Giuliano ${ }^{a}$, Thomas J Slight ${ }^{b}$, \\ Szymon Stanczyk ${ }^{c}$, Szymon Grzankac, Amit Yadav ${ }^{d}$, Kevin E Docherty ${ }^{\mathrm{e}}$, Edik Rafailov, \\ Piotr Perlinc, Stephen P Najda ${ }^{c}$, Mike Leszczynskic, Anthony E Kelly ${ }^{\mathrm{a}}$ \\ ${ }^{a}$ University of Glasgow, School of Engineering, Glasgow, UK, G12 8LT \\ ${ }^{b}$ Compound Semiconductor Technologies Global Ltd, Hamilton, UK, G72 0BN \\ ${ }^{c}$ TopGaN Lasers, Sokolowska 29/37, Warsaw, Poland \\ ${ }^{d}$ Aston University, Birmingham, UK, B4 7ET \\ ${ }^{e}$ Kelvin Nanotechnology Ltd, Glasgow, UK, G12 8LT \\ Tel: (0141) 330 8443, email: s.gwyn.1@research.gla.ac.uk
}

\begin{abstract}
eOver the past 20 years, research into Gallium Nitride (GaN) has evolved from LED lighting to Laser Diodes (LDs), with applications ranging from quantum to medical and into communications. Previously, off-the-shelf GaN LDs have been reported with a view on free space and underwater communications. However, there are applications where the ability to select a single emitted wavelength is highly desirable, namely in atomic clocks or in filtered free-space communications systems. To accomplish this, Distributed Feedback (DFB) geometries are utilised. Due to the complexity of overgrowth steps for buried gratings in III-Nitride material systems, GaN DFBs have a grating etched into the sidewall to ensure single mode operation, with wavelengths ranging from $405 \mathrm{~nm}$ to $435 \mathrm{~nm}$ achieved. The main motivation in developing these devices is for the cooling of strontium ions $\left(\mathrm{Sr}^{+}\right)$in atomic clock applications, but their feasibility for optical communications have also been investigated. Data transmission rates exceeding $1 \mathrm{Gbit} / \mathrm{s}$ have been observed in unfiltered systems, and work is currently ongoing to examine their viability for filtered communications. Ultimately, transmission through Wavelength Division Multiplexing (WDM) or Orthogonal Frequency Division Multiplexing (OFDM) is desired, to ensure that data is communicated more coherently and efficiently. We present results on the characterisation of GaN DFBs, and demonstrate their capability for use in filtered optical communications systems.
\end{abstract}

Keywords: Gallium nitride, optical communications, distributed feedback lasers, filtered communications.

\section{INTRODUCTION}

The development of GaN-based laser diodes (LDs) has been coming to the forefront of semiconductor research for a variety of applications. Initially, GaN LDs were singled out as candidates for lighting, due to their higher output power than standard LEDs, as well as their reduced size and lower energy usage [1]. Such devices are currently used in car headlights [2]. Additionally, the high modulation bandwidth and hence increased data transmission capability that LDs hold over LEDs make them good candidates for free-space or underwater optical communications [3] [4].

For some applications, such as quantum technologies or fluorescence spectroscopy, high spectral purity is extremely important [5] [6], as well as the ability to tune, select, and control a wavelength accurately. To do this, a distributed feedback (DFB) geometry is utilised. Because of the (a) complex overgrowth steps required for a buried grating DFB, which can introduce epitaxial defects, and (b) the dry etch damage induced by etching a surface grating, which may reduce the quality of the p-type contact, a lateral grating etched into the sidewall of the upper cladding close to the active region of the LD is chosen [7].

The potential of such devices when applied to visible light communications (VLC) systems is presented here, with

Fourth International Conference on Applications of Optics and Photonics, edited by

Manuel F. M. Costa, Proc. of SPIE Vol. 11207, 1120700 · ( 2019 SPIE

CCC code: $0277-786 X / 19 / \$ 21 \cdot$ doi: $10.1117 / 12.2527074$

Proc. of SPIE Vol. 11207 1120700-1 
interest in wavelength division multiplexing (WDM) a possible application. GaN DFBs can also be used in underwater communications, as the attenuation coefficient of light through water is lower in the blue and green regions compared to in red/IR [8]. As a consequence of this, using a filtered system to reject background noise would improve system performance, allowing solar rejection. This work exhibits data transmission using a directly modulated, laterally coupled (LC) DFB GaN LD, through measurements such as frequency response, eye diagrams, and bit-error ratio.

\section{DEVICE PROPERTIES}

To enable a single-wavelength $\mathrm{GaN}$ laser to operate, a sidewall grating was etched into (Al,In,Ga) $\mathrm{N}$ based laser epitaxial structures, with fabrication details outlined in [9][10]. For a first-order grating to be realised, feature sizes would be $\sim 40 \mathrm{~nm}$, which is currently impossible due to the technological limitations present in current etching tools. $3^{\text {rd }}$ order gratings are possible however, with features three times larger than those for a $1^{\text {st }}$ order grating are required. To maximise the coupling coefficient, as $3^{\text {rd }}$ order gratings exhibit weaker coupling coefficients compared to their $1^{\text {st }}$-order counterparts, an $80 \%$ duty cycle was implemented for the application of a third order grating [11]. In this work, however, devices based on $39^{\text {th }}$ order notched gratings were used [12], which provide somewhat easier etching methods compared to a previously discussed $3^{\text {rd }}$-order grating device [7] [10]. The main advantage of the higher-order grating geometry is the potential for a much narrower linewidth. This arises due to the fact that a single FP mode is focussed upon with the grating selection method employed. Abdullaev et al. showed that individual FP modes can demonstrate narrower spectral linewidths than conventional DFBs or external cavity diode lasers (ECDL) [13]. Figure 1 shows a scanning electron microscope (SEM) image of the grating.

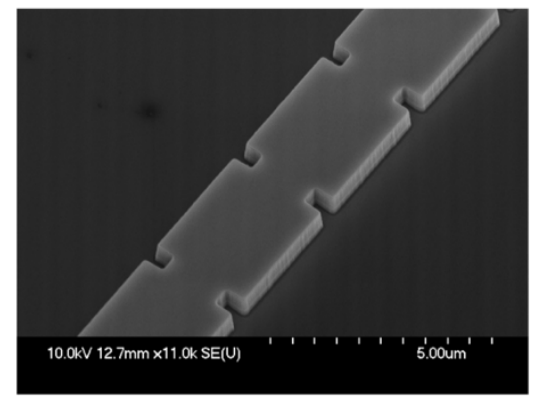

Figure 1. SEM Micrograph image of the $39^{\text {th }}$ order grating.

The devices tested were as-cleaved, uncoated and unpackaged, with a variety of wavelengths, from 405-435nm, fabricated. Figure 2 shows an LVI plot of such a device with a threshold current of $170 \mathrm{~mA}$, and optical output powers of $20 \mathrm{~mW}$ achieved.

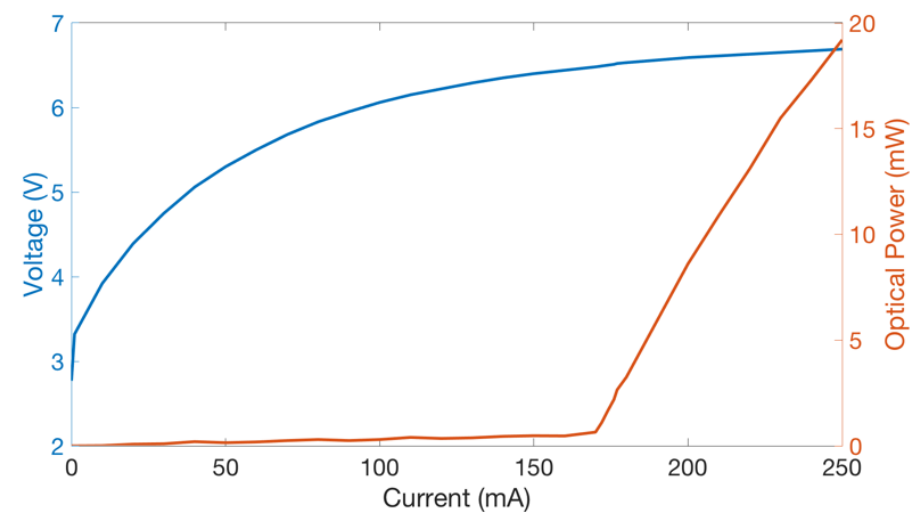

Figure 2. LVI Characteristics of a GaN DFB device.

Spectral measurements were also investigated for various devices, here shown in Figure 3 at $434 \mathrm{~nm}$, with the variations due to (a) current and (b) temperature. As current rises, the device shows an increase of $0.0036 \mathrm{~nm} / \mathrm{mA}$, 
(a)

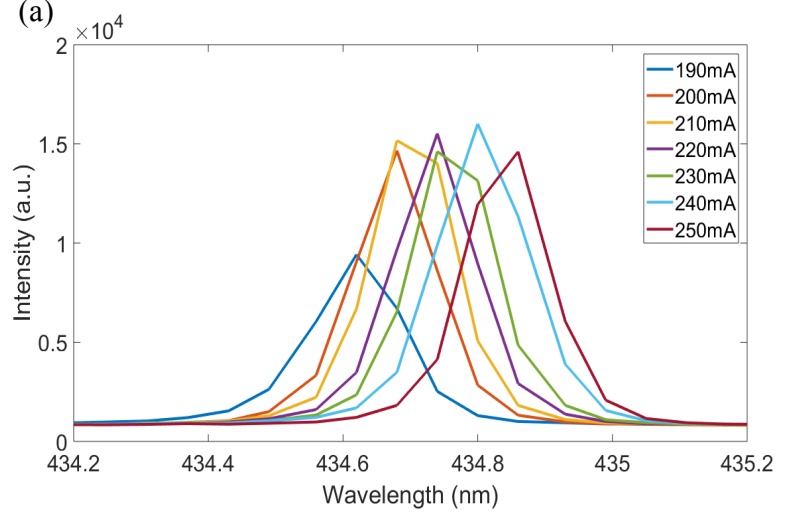

(b)

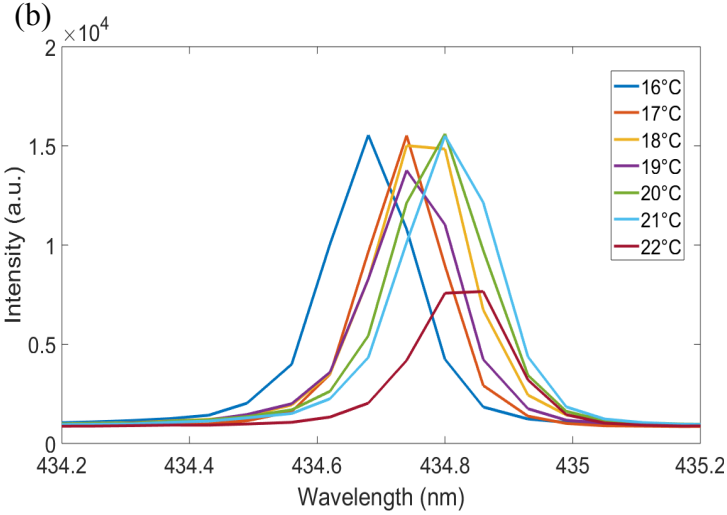

Figure 3. Optical spectra of the GaN DFB at (a) constant temperature with increasing drive currents, and (b) constant drive current and increasing temperature.

while a rate of $0.026 \mathrm{~nm} / \mathrm{K}$ was found when changing temperature. In contrast, a similar GaN FP exhibited a current variation rate of around $0.03 \mathrm{~nm} / \mathrm{mA}$, indicating that the single-wavelength devices are more stable than their FP counterparts by an order of magnitude. The variation in temperature has been researched previously for $10^{\text {th }}$ order surface gratings in [14] [15], with agreement observed in the value of this rate of change.

For applications such as VLC, wavelength stability is extremely important, especially when a WDM system is considered. Precise wavelengths will be necessary in such uses to ensure crosstalk between signals is minimised and that the filtering systems in place would be specific to the exact wavelength of the device. Additionally, in quantum applications such as $\mathrm{Sr}^{+}$atomic clocks, a near-exact wavelength will be required for the cooling transition at $422 \mathrm{~nm}$, and therefore devices that remain stable spectrally will be paramount.

\section{OPTICAL COMMUNICATIONS}

As previously mentioned, single-wavelength GaN LDs have scope for optical communications applications. Because of their spectral stability and precision, these devices would be ideal for filtered communications, where background noise could be rejected and only the laser signal transmitted. Additionally, a WDM setup could be explored, where multiple optical channels are utilised to increase the rate of data transfer. Because of the accuracy of the devices, a system could well be designed with multiple channels transmitting data separated by as little as $1 \mathrm{~nm}$.

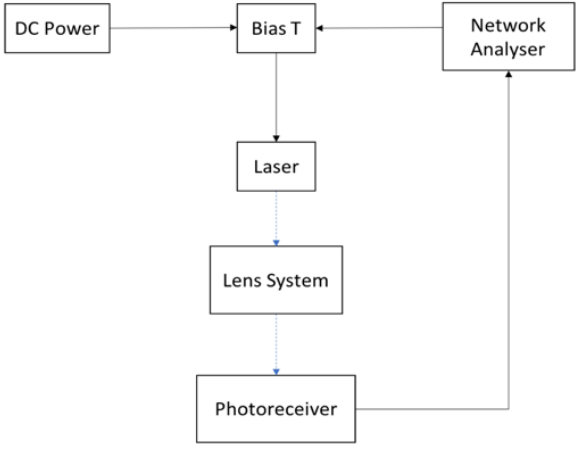

Figure 4. Experimental setup for frequency response measurements.

Firstly, a device as described in section 2 was tested for frequency response to understand modulation capabilities of the devices, with a setup as shown in Figure 4. The optical signal was fed into a Newport 818-BB-21A photoreceiver through to a network analyser. All experiments (apart for variance of wavelength and temperature) 
were undertaken at an operating temperature of $17^{\circ} \mathrm{C}$.

Figure 5(a) shows the frequency response of the $39^{\text {th }}$ order GaN DFB. The maximum recorded bandwidth was observed at $100 \mathrm{~mA}$, which was calculated to be $1.6 \mathrm{GHz}$. Following this, a bandwidth roll-over is apparent, as

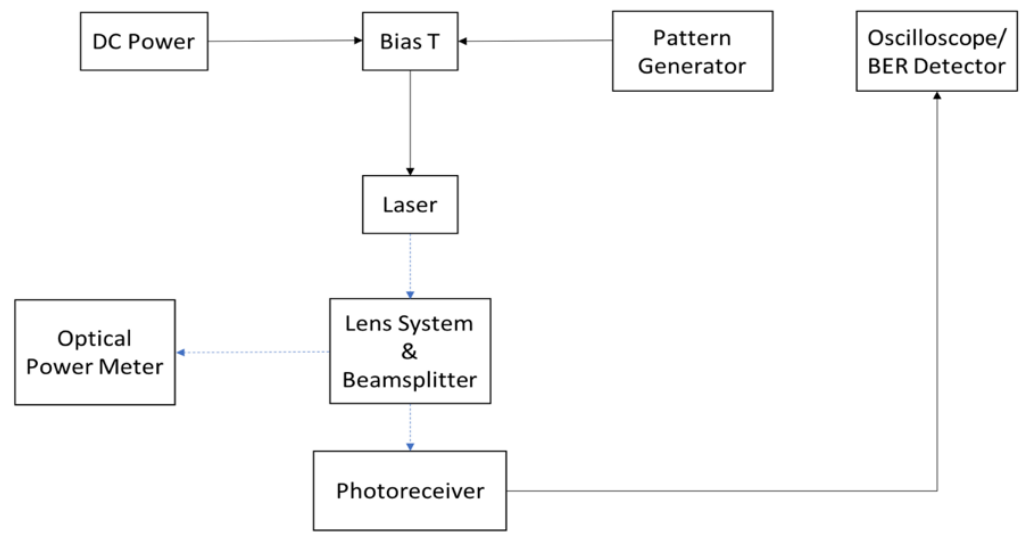

Figure 6. Experimental setup for data rate and BER measurements.

shown in Figure 5(b). This arises from the bandwidth limitations introduced by the photoreceiver.

This was followed by eye diagram measurements and bit-error rate tests, with experimental setup outlined in Figure 6, with the added beamsplitter and optical power meter inserted to record both the error ratio and optical power simultaneously. The same photoreceiver was used here as for the frequency response data collection.

(a)

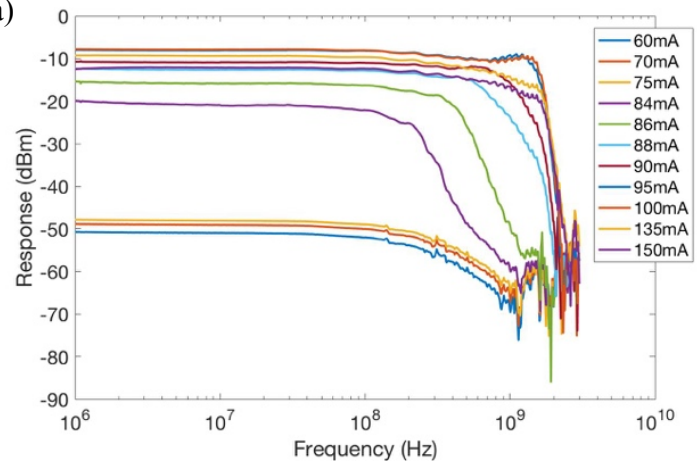

(b)

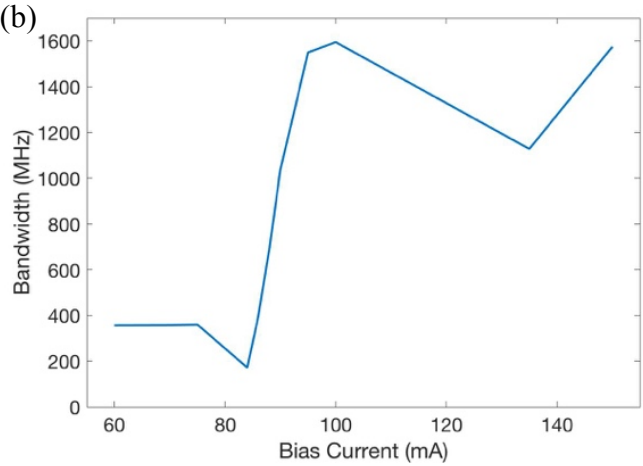

Figure 5. (a) Frequency response of a GaN DFB. (b) Bandwidth of the GaN DFB against bias current.

Eye diagrams were then taken using the same device through the setup outlined in Figure 6, with an oscilloscope connected to the photoreceiver. Even with the restrictions posed by the limited bandwidth of the photoreceiver, error-free data transmission was observed by the device up to $3 \mathrm{Gbit} / \mathrm{s}$, and an open eye diagram was present up to $3.5 \mathrm{Gbit} / \mathrm{s}$. These are shown in Figure 7. 


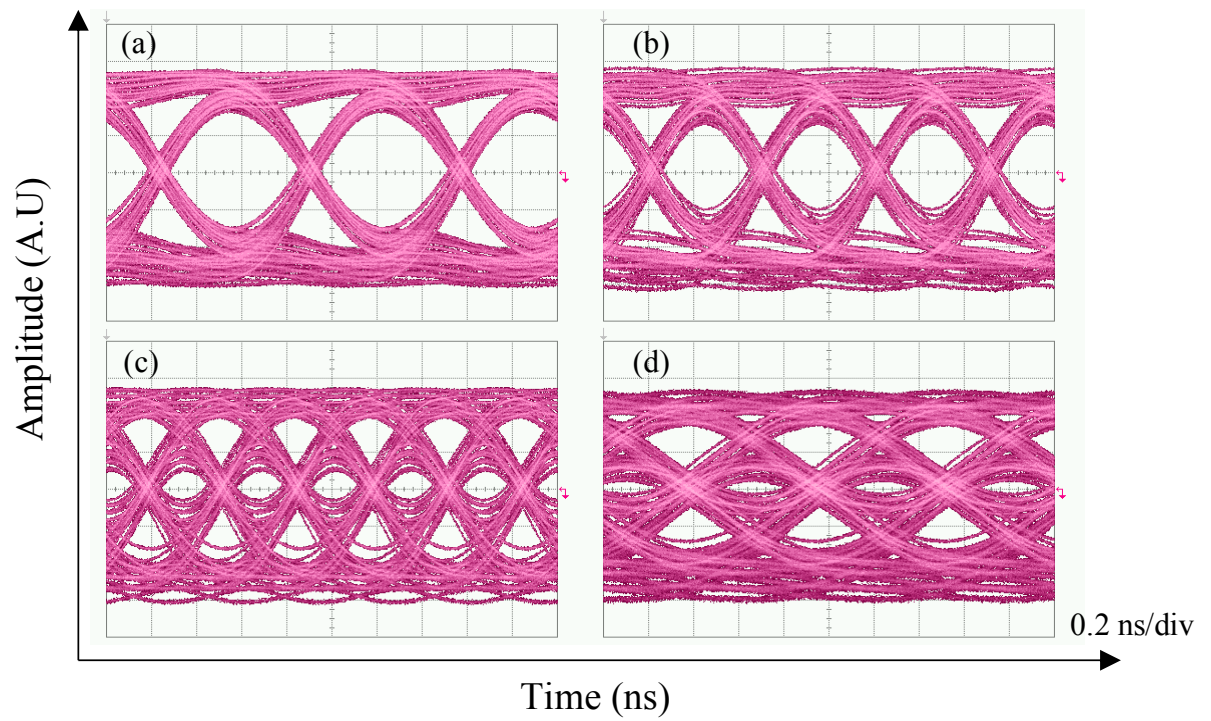

Figure 7. Eye diagrams at (a) $1.5 \mathrm{Gbit} / \mathrm{s}$, (b) $2 \mathrm{Gbit} / \mathrm{s}$, (c) $3 \mathrm{Gbit} / \mathrm{s}$, and (d) $3.5 \mathrm{Gbit} / \mathrm{s}$.

Following this, by replacing the oscilloscope with a bit-error ratio tester (BERT), bit-error rate (BER) measurements could be carried out. Additionally, between the photoreceiver and BERT, a $2 \mathrm{GHz}$ variable gain amplifier (Mini-Circuits ZFL-2000GH+) was inserted, to ensure that error-free transmission was observed at the lowest possible optical power. Figure 8(a) shows the BER for a $407 \mathrm{~nm}$ device at various data transmission rates. A power penalty of $2.1 \mathrm{dBm}$ is observed between $1 \mathrm{Gbit} / \mathrm{s}$ and $2 \mathrm{Gbit} / \mathrm{s}$. This is most likely due to the amplifier's bandwidth being $2 \mathrm{GHz}$, thus the gain exhibited by the device at this data rate is reduced and therefore BER is increased.
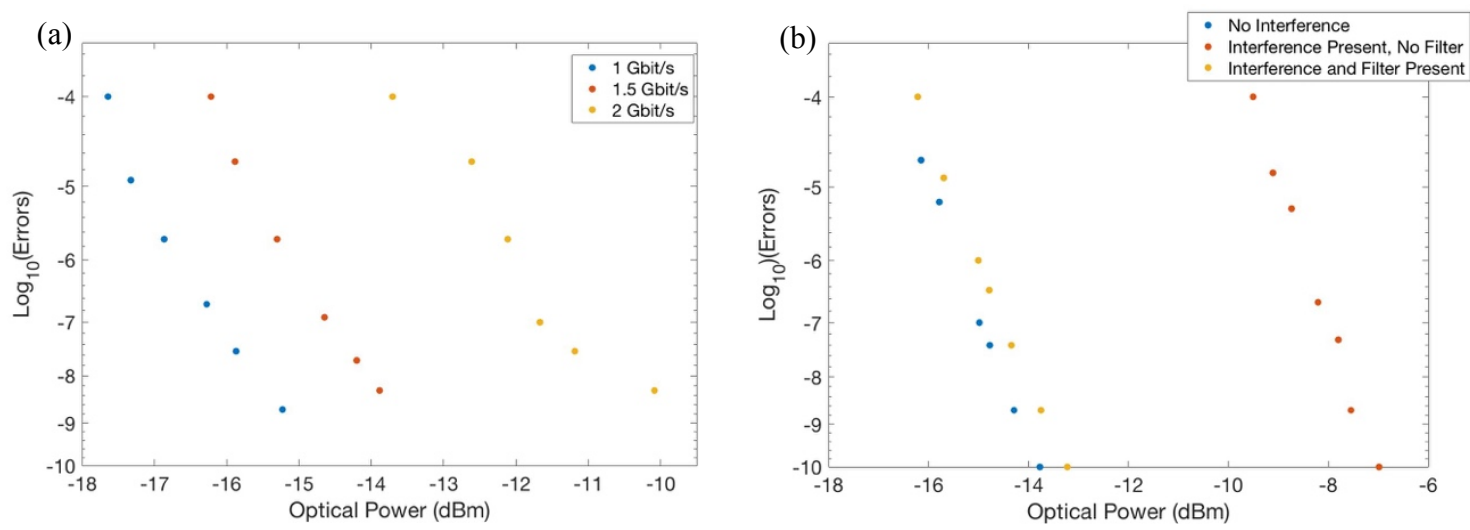

Figure 8. (a) Bit-error rate measurements for a GaN DFB at various data transmission rates. (b) Filtered communications at $1.5 \mathrm{Gbit} / \mathrm{s}$ for the same device.

Finally, by introducing an unmodulated GaN Fabry-Perot (FP) device at a nominal wavelength of $422 \mathrm{~nm}$ into the setup shown in Figure 6, a background signal, or solar noise, was imitated. A $2 \mathrm{~mW}$ interference signal was emitted by the FP. Initially, measurements were taken without any filtering, then a ThorLabs FB405-10 10nm band-pass filter centred at $405 \mathrm{~nm}$ was inserted into the experimental setup. Figure 8(b) shows a $1.5 \mathrm{Gbit} / \mathrm{s}$ signal from the GaN DFB in three different scenarios: without background interference, an interference signal introduced, then adding a filter into the setup. The introduction of the optical filter has effectively neutralised the effect of solar interference, with a power penalty of $0.5 \mathrm{dBm}$ introduced for error-free transmission. With a narrower optical filter, full solar rejection could be achieved. 


\section{CONCLUSIONS}

This work has shown that GaN LDs operating at a precise single wavelength, fabricated in a sidewall grating geometry, are candidates for optical communications applications. Output powers up to $20 \mathrm{~mW}$ have been exhibited, and high stability with current and temperature achieved. Bandwidths up to $1.6 \mathrm{GHz}$ are presented, and error-free data transmission up to $3 \mathrm{Gbit} / \mathrm{s}$. With the introduction of a $10 \mathrm{~nm}$ band-pass filter, partial solar rejection was achieved, and through development of a narrower filtering system total background noise removal would be possible, allowing for WDM systems to be developed.

\section{ACKNOWLEDGEMENTS}

This research has been supported by the European Union with grant E10509, Innovate UK through grant 132543, the National Centre for Research and Development (E10509/29/NCBR/2017 and 1/POLBER-3/2018 and project NCN-2013/11/B/ST3/04263), and the Engineering and Physical Sciences Research Council (RCUK Grant no. $\mathrm{EP} / \mathrm{L} 015323 / 1)$.

\section{REFERENCES}

[1] L. Kuritzky, and J. Speck, "Lighting for the 21st century with laser diodes based on non-basal plane orientations of GaN." MRS Communications, 5(3), 463-473 (2015)

[2] L. Ulrich, "Whiter brights with lasers," IEEE Spectrum, vol. 50, no. 11, pp. $36-56$ (2013)

[3] S. Watson et al., "Visible light communications using a directly modulated $422 \mathrm{~nm}$ GaN laser diode." Opt. Lett. 38, 3792-3794 (2013)

[4] H. M. Oubei et al., "2.3 Gbit/s underwater wireless optical communications using directly modulated 520nm laser diode." Opt. Express 23, 20743-20748 (2015)

[5] S. P. Najda, P. Perlin, T. Suski, L. Marona, S. Stanczyk, P. Wisniewski, S. Grzanka, D. Schiavon, M. Leszczynski, "GaN laser diodes for quantum sensors, clocks and systems," Proc. SPIE 10799, Emerging Imaging and Sensing Technologies for Security and Defence III; and Unmanned Sensors, Systems, and Countermeasures, 107990G (2018)

[6] A. Shiner, "Development of a frequency stabilized 422-nm diode laser system and its application to a $88 \mathrm{Sr}+$ single ion optical frequency standard," no. (2006)

[7] Thomas J. Slight, Opeoluwa Odedina, Wyn Meredith, Kevin E. Docherty, Anthony E. Kelly, "InGaN/GaN DFB laser diodes at $434 \mathrm{~nm}$ with deeply etched sidewall gratings," Proc. SPIE 9748, Gallium Nitride Materials and Devices XI, 97481A (2016)

[8] G Giuliano, L. Laycock, D. Rowe, and A. E. Kelly, "Solar rejection in laser based underwater communication systems," Opt. Express 25, 33066-33077 (2017)

[9] T. J. Slight, O. Odedina, W. Meredith, K. E. Docherty, and A. E. Kelly, "InGaN/GaN Distributed Feedback Laser Diodes With Deeply Etched Sidewall Gratings," IEEE Photonics Technol. Lett., vol. 28, no. 24, pp. 2886-2888 (2016)

[10] Thomas J. Slight et al, "Continuous-wave operation of (Al,In) GaN distributed-feedback laser diodes with high-order notched gratings", Appl. Phys. Express 11 (2018)

[11] Cao, Y.L., Hu, X.N., Luo, X.S., Song, J.F., Cheng, Y., Li, C.M., Liu, C.Y., Wang, H., Tsung-Yang, L., Lo, G.Q. and Wang, Q., "Hybrid III-V/silicon laser with laterally coupled Bragg grating," Optics express, 23(7), 8800-8808 (2015)

[12] Zhong, Y., Zhu, X., Song, G., Huang, Y.., Chen, L., “Two-dimensional simulation of high-order laterallycoupled GaAs-AlGaAs DFB laser diodes," Semicond. Sci. Technol. 19(8), 971-974 (2004)

[13] A. Abdullaev et al., "Linewidth Characterization of Integrable Slotted Single-Mode Lasers," IEEE Photonics Technology Letters, vol. 26, no. 22, pp. 2225-2228 (2014)

[14] J. H. Kang et al., "Optically Pumped DFB Lasers Based on GaN Using 10th-Order Laterally Coupled Surface Gratings," IEEE Photonics Technology Letters, vol. 29, no. 1, pp. 138-141, (2017)

[15] J. H. Kang et al., "DFB Laser Diodes Based on GaN Using 10th Order Laterally Coupled Surface Gratings," IEEE Photonics Technology Letters, vol. 30, no. 3, pp. 231-234, (2018) 